of stations of the Carnegie Institution of Washington is densest in those countries not actually executing magnetic surveys. At various times, comparisons of standards with those of other countries have been obtained, and the establishment of absolute magnetic standards of reference has been greatly improved.

Of the 6,000 stations occupied during some two hundred expeditions on land (1904-40) and upward of 4,000 at sea on the Galilee (1905-8) and the Carnegie (1909-29), at least one third are in the western hemisphere.

The data obtained permit reliable discussions of the distribution of the earth's magnetism and of mysterious variations of its elements from year to year, the so-called magnetic secular variation. These ever-changing lines of magnetic direction and force of the earth, like scientific research, take no account of national boundaries.

Naturally, because of the later opening of new territory in the western hemisphere, Europe at the present time has a great majority of the permanent magnetic observatories. The Americas, however, at the end of the past and during the present century, have established some fifteen permanent observatories well distributed. It would be desirable to have additional observatories at Belem in Brazil and near the southern end of the continent.

Geomagnetic time-changes thus form a main source of information about the physics of the outer layers of the atmosphere, which are subject to distinct cosmical influences on the earth and shield the earth's surface from direct effects. Studies of these outer layers of the atmosphere-the ionosphere-by radio methods supplement magnetic data. Wireless wavepropagation is influenced by the earth's magnetic field, and the location of Huancayo, where the magnetic force is so nearly horizontal, is well suited for experiments on this phenomenon.

The long history of amity and cordial relations between the nations of the western hemisphere supplies a solid foundation upon which in South and North America efforts may be built and co-ordinated for the advancement of earth physics.

\title{
PHYSIOLOGY OF PYRIMIDINES*
}

\author{
By Dr. L. R. Cerecedo, \\ FORDHAM UNIVERSITY
}

$\mathrm{T}$ THREE pyrimidines have been found to occur in Nature as constituents of the nucleoproteins, the chief protein material of the cell nucleus. These pyrimidines are: uracil, thymine and cytosine. Recent investigations on the chemical structure of two vitamins, namely, vitamin $B_{1}$ and riboflavin, have shown that in these substances we are also dealing with pyrimidine derivatives. In spite of the widespread occurrence of the three pyrimidines present in the nureleic acid molecule, very little is known regarding the function of these substances in the animal economy.

The metabolism of uracil, thymine and cytosine has been studied by several investigators, and it has been found that they are catabolized with the conversion of the nitrogen into urea. The intermediate steps in this conversion, however, remained unknown until they were clarified by studies conducted in our laboratory. As a result of these studies, the following scheme for the intermediary metabolism of uracil was suggested : uracil $\rightarrow$ isobarbituric acid $\rightarrow$ isodialuric acid $\rightarrow$ formyloxaluric acid $\rightarrow$ oxaluric acid $\rightarrow$ urea + oxalic acid.

The mechanism suggested by us for the catabolism of uracil has been questioned by Krebs, who studied the action of kidney and liver tissue on uracil, thymine and cytosine. On the basis of his experiments, the details of which have not been reported, Krebs concluded that the pyrimidines were broken down by the kidney, and that the ammonia formed in this process was transformed into urea by the liver.

In view of Krebs's findings, a study of the metabolism of pyrimidines by the tissue slice method has been carried out in our laboratory. The experiments

* Substance of a paper read before Section II (Biological Sciences) of the Eighth American Scientific Congress held in May 1940. involved a study of the breakdown of various pyrimidines by tissue slices. The extent of the metabolism was determined by means of changes in the urea or ammonia content of the medium in which the tissues were suspended. In a series of experiments the effect of pyrimidines on the oxygen uptake of liver tissue was also determined.

In all the experiments there was no significant increase in either urea or ammonia formation in the presence of the pyrimidines investigated. These results seem to indicate that under the conditions used in our experiments there is no marked metabolism of these substances resulting in the formation of ammonia or urea.

The oxygen uptake of mouse liver tissue in the presence of all the pyrimidines studied, and especially in the presence of cytosine, was significantly higher than that of the controls.

The results of this investigation do not eliminate the possibility of other paths of metabolism involving the formation of substances other than ammonia or urea. The validity of the conclusions drawn from the results obtained is determined by the limitations of the tissue slice method. Since waste products cannot be removed, it is necessary to limit the duration of an experiment to a relatively short period. Consequently, if the rate of metabolism is slow, there may not be a sufficiently large accumulation of endproducts to indicate a significant increase. A slow rate of diffusion of the metabolites or end-products would have the same effect. Another limitation of the method is that the cells are removed from their accustomed environment in the animal. Consequently, if the metabolite requires an activator that is not present in the cell itself or in the artificial medium surrounding the cell, metabolism will take place only very slowly and so may escape detection. 